\title{
Expert opinion
}

\section{Prescribing by psychologists?}

Observers of the American Psychological Association's enthusiastic support for the gathering momentum to accredit psychologists with prescribing privileges will detect professional and economic motives among others: the same can also be said of the AMA's campaign to stop it. Health care in the USA is underpinned by a business culture in which psychologists and psychiatrists operate widely as independent practitioners in the open market place. The prestige value and pecuniary advantages to American psychologists would not be insignificant. Also, since health care insurance companies purchase separate psychological and medical treatment for their customers, it would be to their economic advantage for these treatments to be embodied in one individual. Thankfully, pressures on this scale have yet to reach these shores. Nevertheless, serious consideration should be given to establishing a grade of prescribing psychologist in the NHS if it can be shown to raise the overall quality of care; if prescribing could be done appropriately and safely; and if this did not detract from the quality of psychological intervention.

It would be logical for psychologists to have the authority to prescribe only in those instances where the patient needs both psychological and medical intervention (otherwise the debate should be about training more doctors!). The situation is complicated as medical and psychological interventions do not interact uniformly across the range of disorders. There are a number of interventions routinely available under the NHS which are primarily psychological where medication largely serves a supportive function, for example the management of panic and obsessive/compulsive disorders: in other cases psychological and medical interventions are known to be additive, for example the use of cognitive therapy and tricyclics in some forms of depression; and there are those which are predominantly medical, the use of neuroleptics in acute psychosis being the obvious example. There can be little sense in psychologists having a purely prescribing function as in the latter example; there are barely enough psychologists to deliver psychological treatments let alone medical ones. I think a case might be made in respect of those interventions which are psychologically led, that the quality of the overall service to the patient would be increased if a single practitioner co-ordinated both psychological and drug treatments. This would enable a more informed and prompt titration of medication with the psychological treatment according to the overall progress of the patient. I think this would meet with patients' approval, many of whom are confused by the practice of dual medical and psychological management. Managers also might find such a prospect attractive since it would reduce the amount of service contact per patient without prejudicing the quality of care.

For many, however, the issue will rest on whether psychologists can be trained to prescribe appropriately and safely. Under limited circumstances I have little doubt that this could be achieved. In general, the greater the independence afforded in prescribing the more the training of psychologists would have to approximate to some aspects of that of psychiatrists; however with sufficient training and a system of supervision a degree of 'devolved' prescribing could be considered for those limited situations described above. Any such initiative would require a considerable investment in training, which must be weighed in the balance against time taken away from psychological training, in addition to consideration of the medico-legal implications.

It is instructive to note that a parallel issue has been debated in clinical psychology: namely should psychological treatment methods be disseminated to non-psychologists? Very similar issues have been raised, most notably the quality of intervention and the possible harm of that can be done to patients if these skills are placed in the hands of poorly trained therapists lacking the appropriate theoretical background. It has recently been recommended (MPAG, 1990) that dissemination become a formal, recognised function of the profession in the health service. The report draws upon a distinction between levels of psychological skill required to undertake different kinds of therapeutic activity. There are those circumscribed activities for which the therapist requires specific training and supervision; these are distinguished from activities which require specialist skills in circumstances where there are deep rooted underlying influences which call for the capacity to draw on multiple theoretical models to devise an individually tailored strategy for a complicated presenting problem. A similar distinction in terms of levels of prescribing skill may be helpful to this debate. 
The foregoing includes some of the issues which might inform a debate about whether we should follow the lead set by the Americans. Try as I might to argue the case for the prescribing psychologist, my heart is not really in it. My over-riding concern is that such a move could ultimately lead to pressures that would jeopardise the quality of psychological treatments. Psychologists are trained and employed to ease distress through psychological means; this is both a pressure and a challenge which has stimulated innovation. In the context of service pressure in the NHS, the temptation to prescribe as an expedient will always be present, and will detract from attention given to patients' psychological needs. The developing structure of health care in the NHS needs to be considered too. The multidisciplinary model of service provision (e.g. through community mental health teams) is becoming the dominant one in the UK and its strength lies in the accessibility of the specialist skills of a range of professionals. In the writer's view, there comes a point at which the blurring of roles and the sharing of skills ceases to become a means of strengthening multidisciplinary team work but a recipe for mediocrity. The concept of a prescribing psychologist would be a case in point.

Clinical Psychologist

All Saints Hospital

Birmingham B18 5SD

Max BiRCHWOOD

\section{Reference}

Manpower Planning Advisory Group (1990) Clinical Psychology Project, Department of Health.

It seems there's a drive on in the US to allow clinical psychologists at least limited prescribing rights. According to the BMJ (18-25 August 1990, p. 356) those working in Reservations have been able to prescribe drugs to Indians on their own initiative in recent years, and now the US Department of Defense has a training programme in psychopharmacology for two psychologists so they can give patients diazepam, haloperidol, chlorpromazine and benztropine. Apparently some States already grant legal prescribing rights to dentists, nurses and other paramedics in certain circumstances. The American Psychological Association is favourable, and government is being prodded to take positive action.

Psychologists are cheaper than doctors to employ, have a shorter university training, may be easier to recruit for some services; and therefore are likely to appeal strongly to the government mind. On the other hand, in Britain since 1858 the law has limited the prescribing of a whole range of drugs to those on the Medical Register, the idea being that using drugs properly is a skilled business and should be in the hands of people with a standard wide training. This means acquaintance not only with physiology, biochemistry and pharmacology but with the sort of side effects and changes produced in different organs, which may mimic diseases; and nowadays a considerable amount of illness is iatrogenic. The doctor learns a good deal about this in the wards during student and intern years; practical experience influenced by fellow housemen and by experienced seniors. Medicine is still taught partly through apprenticeship. If clinical psychologists are to be taught the use of even a few drugs they will need not merely lectures but some months at least of supervised experience before they can be let loose on their own, and it is hard to believe they will manage as well as someone who has had a fuller and wider training.

Giving a dose of salts, or aspirin for headache, or a five-day course of antibiotic capsules has a simplicity about it which does not apply through the whole range of therapeutics. Chlorpromazine is not only anti-psychotic but provokes neurological disorder. It acts not only on the brain but on the liver, the kidney, the blood, the skin, and interacts with other medicines the patient may be taking. A wide clinical awareness is necessary in using it. Its prescription is not just a matter of writing doses and times on a piece of paper, but of follow-up and variations over periods of weeks and months. Is this really work for someone whose interests and other training lie elsewhere? Is a third-class service good enough for Indians, the unemployed, the chronic psychotics?

Of course the service we offer now is often second class. Psychiatrists and GPs do not always prescribe the right drugs in the right doses to get the best results possible, and there are iatrogenic illnesses for which we must admit responsibility. Psychiatric treatment is a difficult art. We do not always take enough thought and care, or know all we might, and our attention is often on psychotherapy or social therapy instead of on the drugs. Perhaps we need the backup of psychiatric chemotherapy as a recognised subspeciality, and ought to be prepared to refer cases to the chemical therapist for medical treatment as we use the psychotherapist. The lithium clinic and depot clinic are steps this way. Under supervision a psychologist might play a useful role here.

J. L. Crammer

Emeritus Reader in Biological Psychiatry

Institute of Psychiatry

London SE5 8AF 